Article

\title{
Evaluation Model of Aluminum Alloy Welded Joint Low-Cycle Fatigue Data Based on Information Entropy
}

\author{
Yaliang Liu ${ }^{1,2}$, Li Zou ${ }^{1,2}$, Yibo Sun ${ }^{1,2}$ and Xinhua Yang ${ }^{1,2, *}$ \\ 1 School of Material Science and Engineering, Dalian Jiaotong University, Dalian 116028, China; \\ Liuyaliang2009@126.com (Y.L.); lizou@djtu.edu.cn (L.Z.); yibo_sun@126.com (Y.S.) \\ 2 Dalian Key Laboratory of Welded Structures and Its Intelligent Manufacturing Technology (IMT) of Rail \\ Transportation Equipment, Dalian Jiaotong University, Dalian 116028, China \\ * Correspondence: yangxhdl@gmail.com; Tel.: +86-411-8410-5956
}

Academic Editor: Raúl Alcaraz Martínez

Received: 8 December 2016; Accepted: 16 January 2017; Published: 18 January 2017

\begin{abstract}
An evaluation model of aluminum alloy welded joint low-cycle fatigue data based on information entropy is proposed. Through calculating and analyzing the information entropy of decision attributes, quantitative contribution of stress concentration, plate thickness, and loading mode to the fatigue destruction are researched. Results reveal that the total information entropy of the fatigue data based on nominal stress, structural stress and equivalent structural stress are, respectively, $0.9702,0.8881$, and 0.8294 . There is consistency between the reducing trend of the weight-based information entropy and the smaller and smaller standard deviation of the S-N curves. In the structural stress based S-N curve, total stress concentration factor is crucial for the distribution of the fatigue data and the weight based information entropy of membrane stress concentration factor is 0.6754 , which illustrates that stress concentration is a key issue of welded structure to which ought to be attached great importance. Subsequently, in the equivalent structural stress-based S-N curve, the weight based information entropy of stress ratio is 0.5759 , which plays an important role in the distribution of fatigue data. With the importance level of the attributes on the S-N curves investigated, the correction of $R$ in the equivalent structural stress based master S-N curve method should be carried out to make the welding fatigue prediction much more accurate.
\end{abstract}

Keywords: fatigue; information entropy; equivalent structural stress; welded joints

\section{Introduction}

At present, the widely-applied welding fatigue design and prediction are mainly based on nominal stress (NS) approach, hot spot stress (HSS) approach, and the master S-N curve approach [1]. As is well-known, the nominal stress method was firstly proposed and used maturely for the fatigue analysis of welded structures [2]. Due to the fact that it ignores the local stress effects of welded joints and is explicitly mathematically explained, many standard specifications have been established based on the nominal stress method using a large scale of experimental data from [3,4]. While some limitations have gradually come to light, the nominal stress of a complex structure is hard to determine and inconsistent stress calculation is caused by a singularity in the weld toe as well, which limit its universality in engineering applications [5]. Afterwards, in order to obtain the accurate stress in the welding toe, the hot spot stress (HSS) method that is extrapolated based on the nominal stress was first proposed by Niemi [6]. It characterizes the stress condition in the weld toe by taking the effects of geometry structure into account so that hot spot stress can be extrapolated through an extrapolation formula indirectly in complex welded structures [7]. Although this solves some problems existing in the nominal stress method, it is difficult to establish a uniform hot spot type and extrapolation formula for various welding types, which also limits its engineering applications. 
As discussed in [1], an innovative master S-N curve approach based on the equivalent structural stress (ES) has been proposed by Dong via taking advantage of the new concept of structural stress (SS). The effectiveness of the master S-N curve approach is attributed to the fact that the structural stress calculated are mesh-insensitive and, therefore, intrinsic to a given joint geometry and loading mode from the perspective of fracture mechanics. Thus, an equivalent structural stress parameter in the context of a single master S-N curve has been established and proven to be effective in consolidating a large amount of weld S-N data obtained from drastically different joint geometries, plate thickness, and loading mode [8].

Information entropy was defined by Shannon, "The father of information theory", which can measure the uncertainty of random events. Originally, the concept of information entropy was used to measure the dispersion degree. Information entropy has been widely used in many field, such as engineering technology, economic decision-making, societal risk, etc. [9-15].Currently, information entropy is gradually being used in the field of welding and fatigue research. Li et al. [9] combined the theory of wavelet multi-resolution analysis with the information entropy together and proposed an algorithmic method of wavelet energy entropy based on wavelet multi-resolution analysis so that the wavelet energy entropy can be used to evaluate the stability of arc in square wave alternating current submerged arc welding. Kavrishvili et al. [10] developed a method for the evaluation of the information content of the signals of welding current and arc voltage taking into account the statistical dependence of the appearance of the values in the given time range. Results show that these quantitative characteristics may be used for the evaluation of the quality of the characteristics of the components of the welding process. The concept of thermodynamic entropy generation was employed by Amiri et al. [11] to assess degradation in processes involving metal fatigue. Conclusions were carried out that empirical fatigue models such as Miner's rule, Coffin-Manson equation, and Paris law can be deduced from thermodynamic consideration. In addition, with the help of information entropy under multi-step independent communications from many sources, Skorobogatov [12] described the disclosing indeterminacy in a pattern of cracks from the beginning and up to the end of loading for the first time. The curve of the parameter of serviceability coincides with the curve of the information entropy by the character of change which determines an assessment of expedience rehabilitation of a structure's damage with unknown load. Incorporating all of the uncertainties, a maximum relative entropy (MRE) approach is proposed and developed by Guan et al. [13] to update the statistical description of model parameters and narrow down the prognosis deviations. A detailed comparison between the proposed MRE approach and the classical Bayesian updating method is performed to illustrate advantages of the proposed prognosis framework. Additionally, a general method of probabilistic fatigue damage prognosis using limited and partial information is developed [14]. The posterior distribution is formulated using the principle of maximum relative entropy (MRE) to perform probability updating is point measurements. Fatigue crack prognosis problems with experimental data are presented for demonstration and validation. The maximum entropy fracture model [15] is a thermodynamically consistent and information theory inspired (non-empirical) damage accumulation theory for ductile solids, validated on both area array and peripheral array packages. Combining the authors' knowledge of the constitutive behavior with the damage accumulation behavior, fatigue life predictions can be made for a wide variety of package types and mixed metallurgical conditions. A computational method based on the maximum entropy principle is proposed by Li et al. [16] for identifying the probability distribution of fatigue life. The rationality and effectiveness of the proposed method are demonstrated by two groups of fatigue datasets available in the literature. Comparisons among the proposed method, the lognormal distribution and the three-parameter Weibull distribution are also carried out for the investigated groups of fatigue datasets.

In this paper information entropy was firstly employed to analyze the S-N data distribution of welding structure based on the equivalent structural stress. Probability distribution is analyzed by the proposed model, and importance level of the attributes governing the welding structure fatigue life is investigated for the first time by entropy ideology. Firstly, three S-N curves for aluminum alloy 
welded joints are established based on nominal stress, structural stress, and equivalent structural stress in the master S-N curve method. Then an evaluation of fatigue life data based on information entropy is established to study the correction of S-N distribution of the three kinds of approaches. There is consistency between the reducing trend of the weight based information entropy and the smaller and smaller standard deviation of the S-N curves. Especially, through calculating and analyzing the weighted information entropy of decision attributes, quantitative contribution of stress concentration, plate thickness and loading mode to the fatigue destruction are studied. Thus, the fatigue life evaluation method based on the information entropy can be a credible and effective method of the weld fatigue prediction.

\section{Basic Theory}

\subsection{Theory of Equivalent Structural Stress}

When the stress of welding structure is calculated by the means of finite element method, mesh size has significant effects on stress distribution especially at the zone of stress concentration, which is detrimental to predict the fatigue life accurately. From the fracture mechanics point of view, weld toe fatigue cracks should be dominated by the through-thickness nonlinear stress distribution perpendicular to the crack plane in the base plate at the failure location. At this point, a simple welded T-joint subjected to a tension load $\mathrm{P}$ of $\mathrm{X}$ direction is taken as an example to illustrate the through-thickness nonlinear stress distribution as shown in Figure 1.

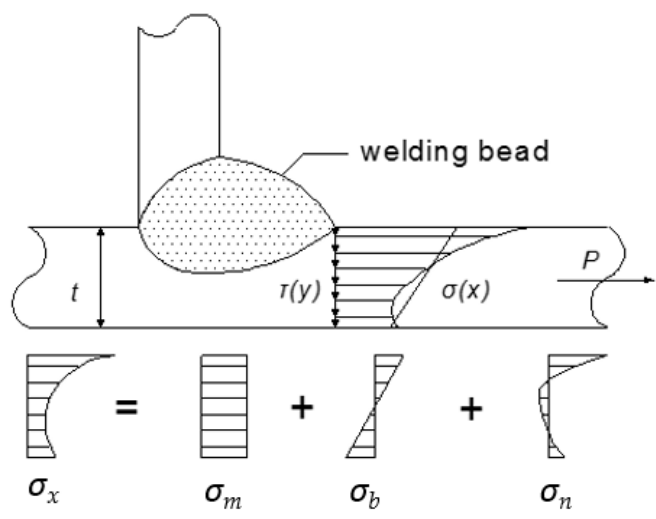

Figure 1. Through-thickness nonlinear stress distribution on welding toe.

An accurate measure of the through-thickness stress distribution is to characterize any through-thickness stress state in the form of three parts: membrane stress $\left(\sigma_{m}\right)$, bending stress $\left(\sigma_{b}\right)$, and nonlinear peak stress $\left(\sigma_{n}\right)$. While the nonlinear peak stress is self-equilibrium so that it can be ignored. Therefore the through-thickness stress on welding toe can be simplified into the combination of $\sigma_{m}$ and $\sigma_{b}$. The former characterizes the through-thickness uniform part of the stress state and the latter characterizes the linear part of the stress gradient in the stress state. The normal structural stress based on the line force at each node from elementary structural mechanics theory is given by:

$$
\begin{gathered}
\sigma_{s}=\sigma_{m}+\sigma_{b} \\
\sigma_{m}=\frac{F_{y}}{A}=\frac{F_{y}}{l \cdot t}
\end{gathered}
$$




$$
\begin{gathered}
\sigma_{b}=\frac{M_{x}}{W}=\frac{M_{x}}{\frac{1}{6} \cdot l \cdot t^{2}} \\
\sigma_{s}=\sigma_{m}+\sigma_{b}=\frac{f_{y}}{t}+\frac{6 m_{x}}{t^{2}}
\end{gathered}
$$

where $F_{y}$ is the force vertical to the weld toe, $A$ is the sectional area of the plate, $l$ is the width of the plate, $t$ is the thickness of the plate, $M_{x}$ is the moment around the weld toe, $W$ is the moment of inertia around the weld toe, $f_{y}$ is the corresponding line force vertical to the weld toe, and $m_{x}$ is the corresponding line moment around the weld toe. Therefore, structural stress (SS) $\sigma_{S}$ at each node can be defined as above [1].

By introducing the Paris crack growth law Equation (5), the stress intensity factor of crack propagation theory can be calculated as [17]:

$$
\begin{gathered}
\frac{d a}{d N}=C \cdot M_{k n}^{n}\left(\frac{\Delta K}{\sqrt{1-R}}\right)^{m} \\
\Delta K=\Delta K_{m}+\Delta K_{b}=\frac{\sqrt{t^{*}}}{1-R}\left[\Delta \sigma_{m} f_{m}\left(\frac{a}{t}\right)+\Delta \sigma_{b} f_{b}\left(\frac{a}{t}\right)\right]
\end{gathered}
$$

where $a$ is the crack depth, $N$ is number of cycles, $C$ is crack growth constant, $M_{k n}$ is the notch stress magnification expressed as $M_{k n}=K / K_{n}$ in which $K$ represents the total $K$ due to both the far-field stress and the local notch stress effects and $K_{n}$ represents only the far-stress contribution to the stress intensity factor $n$ is crack growth exponent in the short crack regime, $m$ is crack growth exponent in the long crack regime, $R$ is stress ratio, $t^{*}$ is interpreted as a ratio of actual thickness $t$ to a unit thickness, rendering the term dimensionless. The parameters $f_{m}(a / t)$ and $f_{b}(a / t)$ are well-known dimensionless functions of $a / t$ corresponding to the membrane and bending components. By simultaneous combination of Equations (5) and (6), the prediction of the lifecycle from an infinitesimally small crack to final failure can be expressed as:

$$
N=\int_{a / t=0}^{a / t=1} \frac{t^{*} d(a / t)}{C\left(M_{k n}\right)^{n}\left(\frac{\Delta K}{\sqrt{1-R}}\right)^{m}}=\frac{1}{C} t^{*\left(1-\frac{m}{2}\right)}\left(\Delta \sigma_{s}\right)^{-m} I(r)(1-R)
$$

where $I(r)$ is a dimensionless function of $r$ and $m$ is the crack growth exponent, which is set to be 3.6 in ASME [18]. A master S-N curve can be established according to Equation (7) based on a set of welding fatigue data. The equivalent structural stress related to Equation (7) can be expressed as:

$$
\Delta S_{s}=\frac{\Delta \sigma_{s}}{(1-R)^{\frac{1}{m}} \cdot I(r)^{\frac{1}{m}} \cdot t^{\frac{2-m}{2 m}}}
$$

\subsection{Basic Principle of Information Entropy}

Information entropy that can be a measure of uncertainty is a probabilistic concept. According to the characteristics of entropy, the randomness and dispersion of an event can be determined by calculating the information entropy. The greater the value of the information entropy is, the more effects factors have on the comprehensive evaluation [19]. In this paper the information entropy was used to analyze the S-N distribution based on the nominal stress, structural stress and equivalent structural stress, through which a brand new evaluation criteria based on information entropy was established to estimate the welding fatigue prediction approaches.

During the process of information calculation, there are some concepts based on the following considerations: 
a. It is assumed that there are $n$ factors $X_{1}, X_{2}, X_{3} \ldots X_{n}$ related to an event, the values of which make up the decision-making matrix $D$ as follows:

$$
D=\left[\begin{array}{cccc}
d_{11} & d_{12} & \ldots & d_{1 n} \\
d_{21} & d_{22} & \ldots & d_{2 n} \\
\ldots & \ldots & \ldots & \ldots \\
d_{m 1} & d_{m 2} & \ldots & d_{m n}
\end{array}\right]
$$

b. Firstly in order to obtain the weight of each factor later, it is necessary to transform the decision-making matrix $D$ to decision-making matrix $R$ by normalization:

$$
R=\left[\begin{array}{cccc}
r_{11} & r_{12} & \ldots & r_{1 n} \\
r_{21} & r_{22} & \ldots & r_{2 n} \\
\ldots & \ldots & \ldots & \ldots \\
r_{m 1} & r_{m 2} & \ldots & r_{m n}
\end{array}\right]
$$

Along this line, the sum of each column element equals 1, or in other words the decision-making matrix $R$ satisfy the equation:

$$
\sum_{i=1}^{m} r_{i j}=1, j=1,2,3 \ldots, n
$$

c. The column vectors $\left(A_{1}, A_{2} \ldots A_{n}\right)$ of the normalized decision-making matrix $R$, namely the factors value $\left(r_{1 j}, r_{2 j} \ldots r_{m j}\right)$ of the factors $\left(X_{1}, X_{2} \ldots X_{n}\right)$ are treated as a probabilistic distribution of information. The information entropy $E_{j}$ of the factor $X_{j}$ is defined as:

$$
E_{j}=-\frac{1}{\ln m} \sum_{i=1}^{m} r_{i j} \ln r_{i j}, j=1,2,3 \ldots, n
$$

Here it is not difficult to understand that $0 \leq E_{j} \leq 1$.

\section{Fatigue Data Evaluation Mode Based on Information Entropy}

\subsection{Establishment of the S-N Curve}

As introduced before, information entropy was employed to analyze the S-N data distribution of welding structure based on the three types of stress in this paper. Firstly three S-N curves for aluminum alloy welded joints are established based on nominal stress, structural stress, and equivalent structural stress by means of the least square method. Then an evaluation mode of fatigue life data based on information entropy is established to study the correction of S-N distribution of the three types of approaches. Furthermore, the contrast between the weight based information entropy and the standard deviation of the S-N curves were carried out. Through calculating and analyzing the weighted information entropy of decision attributes, quantitative contribution of stress concentration, plate thickness and loading mode to the fatigue destruction are studied.

It is worth noting here that all of the S-N curves modeled and information entropy calculated in this paper are based on well-documented fatigue data. Aluminum alloy low cycle fatigue data for MIG [19], GMAW [20], TIG [21], manual arc [22], and in the as-welded conditions without stress-relief were cited. Forty-eight sets of welding fatigue data were arranged by welding factors including stress type $(T)$, plate thickness $(t)$, load ratio $(R)$, and stress ratio $(r)$, among which the parameters $R$ and $r$ are expressed as: 


$$
\begin{gathered}
r=\frac{\sigma_{b}}{\sigma_{m}+\sigma_{b}} \\
R=\frac{\sigma_{\min }}{\sigma_{\max }}
\end{gathered}
$$

With respect to these data, the mean S-N curves based on NS, SS, and ES were fitted using the least square method and the standard deviations (StaDev) were calculated at the same time. The mean S-N curves and deviation curves with $-3,-2,-1,+1,+2$, and +3 magnifications were then carried out in respective coordinates, as shown in Figures 2-4 [5]. According to the parameters above, the S-N data points based on NS, SS, and ES, respectively, were discretized as shown in Figures 2-4. It is necessary to explain here that there are different experiment parameters as shown in Table 1 from Joint-A to Joint-E.

Table 1. Experiment parameters of the well-documented fatigue data.

\begin{tabular}{cccccc}
\hline Category & Joint-A & Joint-B & Joint-C & Joint-D & Joint-E \\
\hline Joint Type & TJ:p & TJ:p & LJ_SS:p & LJ_DS:p & SJ_DS:p \\
Load Type & $4 \mathrm{~B}$ & $4 \mathrm{~B}$ & $\mathrm{~T}$ & $\mathrm{~T}$ & $\mathrm{~T}$ \\
$\mathrm{R}$ & 0.1 & 0.5 & 0.1 & 0 & 0 \\
$\mathrm{t}$ & 10 & 10 & 2.5 & 3 & 4.7625 \\
$\mathrm{r}$ & 0.998243 & 0.998243 & 0.750616 & 0.655401 & 0.344753 \\
SCFm & 0.00188 & 0.00188 & 1.0012 & 1.01809 & 1.43617 \\
SCFb & 1.06822 & 1.06822 & 3.0135 & 1.93633 & 0.75563 \\
SCF & 1.0701 & 1.0701 & 4.0147 & 2.95442 & 2.1918 \\
Welding Procedure & MIG & MIG & GMAW & TIG & Manual Arc \\
\hline
\end{tabular}

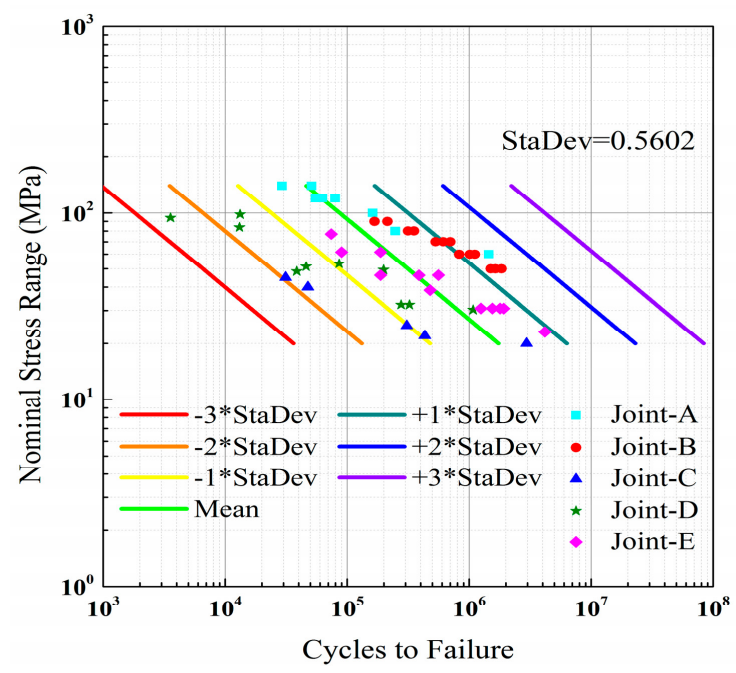

Figure 2. S-N distribution based on nominal stress. 


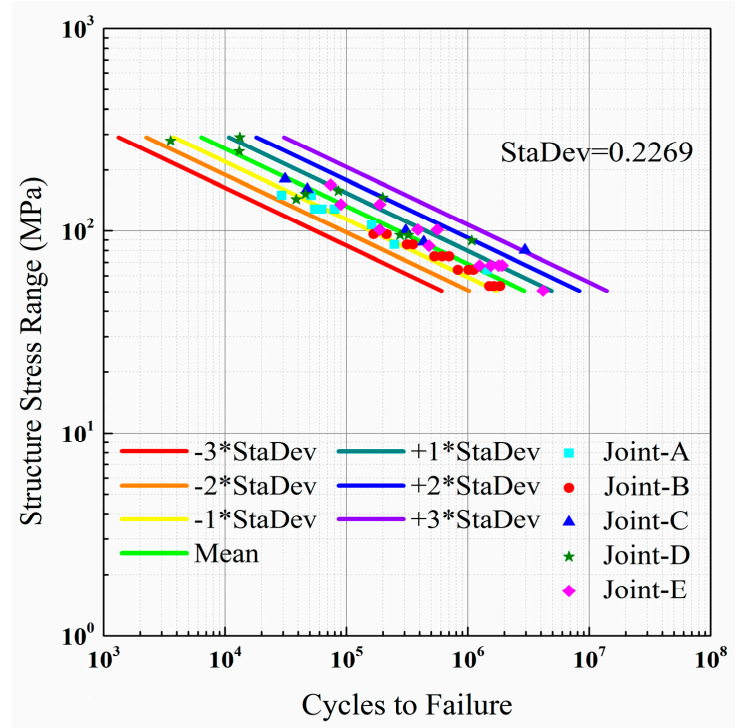

Figure 3. S-N distribution based on structural stress.

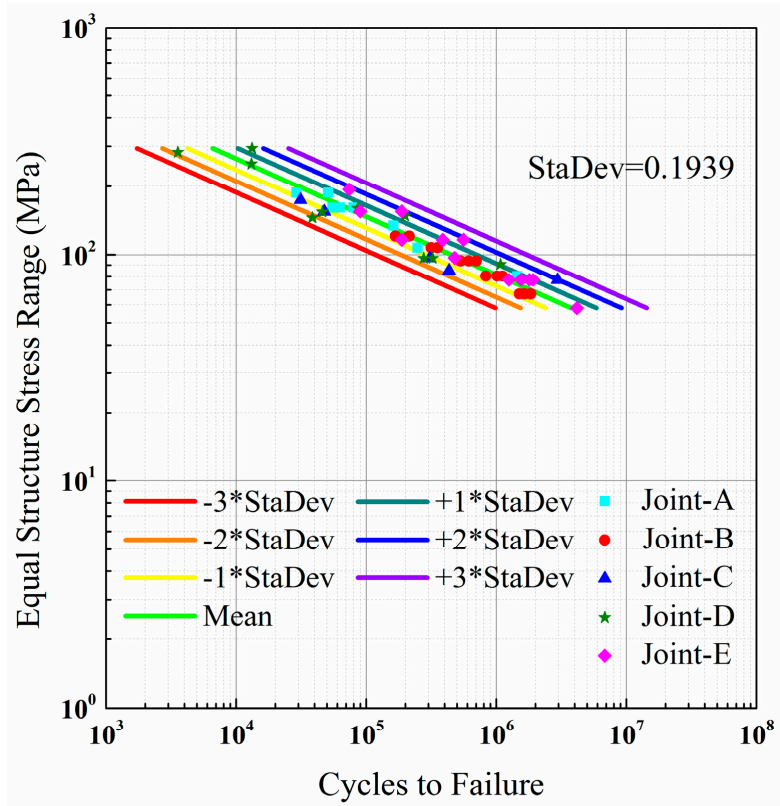

Figure 4. S-N distribution based on equivalent structural stress.

In order to analyze the distribution and dispersion of the fatigue data contrastively, for all of the S-N curves above, the scale of the horizontal axis is from $10^{3} \mathrm{~N}$ to $10^{8} \mathrm{~N}$ as well as the scale of the vertical axis is from $10^{0} \mathrm{MPa}$ to $10^{3} \mathrm{MPa}$. The standard deviation of the three S-N curves is $0.5602,0.2269$, and 0.1939 , respectively, and it is obvious that the width between the deviation curves is narrower, which is consistent with the smaller and smaller standard deviation. As illustrated above, the data points of the S-N curve based on the nominal stress scatter between the -3 and +2 magnifications deviation curves. The scale of the deviation curves is broad and the standard deviation is 0.5602 , the largest of all. The structural stress rearranges the S-N discretized points, so that they are much more concentrated in the field nearby the mean S-N curve and the standard deviation becomes 0.2269 which is much smaller than the former. The equivalent structural stress translates them more uniform on both sides of the mean S-N curve and the smallest standard deviation 0.1939 is obtained. With the correction step the concentration and uniformity of S-N distribution were enhanced, which creates a Mmaster S-N curve with a single narrow brand curve. 


\subsection{Fatigue Data Evaluation Model Based on Information Entropy}

In terms of decision-making, the concept of information entropy is usually introduced to determine the weight of attributes, according to which the greater the information entropy is, the more effects the attributes have on the dispersion degree. In addition, according to Dong [8], all kinds of attributes have different level of effects on the concentration degree of the S-N distribution. Thus, from the weight point of view, the total information entropy of the attributes governing the welding fatigue was calculated and analyzed. Eventually, a brand-new evaluation procedure of welding fatigue was obtained.

In order to take advantage of information entropy to ascertain the weight of the attributes and then establish fatigue data evaluation model, information entropy calculation procedure of equivalent structural stress, for instance, is addressed according to the well-documented data [16-19], which is shown in Table 2.

Table 2. Well-documented fatigue data.

\begin{tabular}{cccccccccc}
\hline No. & $N S$ & $S S$ & $E S$ & $S C F_{m}$ & $S C F_{b}$ & $S C F$ & $R$ & $t$ & $r$ \\
\hline 1 & 140 & 149.814 & 187.699 & 0.00188 & 1.06822 & 1.0701 & 0.1 & 10 & 0.99824 \\
2 & 80 & 85.608 & 107.257 & 0.00188 & 1.06822 & 1.0701 & 0.5 & 10 & 0.99824 \\
3 & 40 & 160.588 & 154.714 & 1.0012 & 3.0135 & 4.0147 & 0.1 & 2.5 & 0.75062 \\
$\ldots$ & $\ldots$ & $\ldots$ & $\ldots$ & $\ldots$ & $\ldots$ & $\ldots$ & $\ldots$ & $\ldots$ & $\ldots$ \\
48 & 94.288 & 278.566 & 282.502 & 1.01809 & 1.93633 & 2.95442 & 0 & 3 & 0.65540 \\
\hline
\end{tabular}

According to Equation (8), the value of ES is determined by the following four parameters and the effects of stress concentration factor (SCF) has been taken into account in the SS method. Thus, on the basis of Equations (9)-(12), information entropy of the four attributes structural stress (SS), stress ratio $(R)$, plate thickness $(t)$, and load ratio $(r)$ is $0.9735,0.7790,0.9667$, and 0.9819 , respectively.

Next the distinction degree $F_{j}$ of $X_{j}$ is defined as:

$$
F_{j}=1-E_{j}
$$

Hence, distinction degree of the four attributes $S S, R, t$ and $r$ is $0.0265,0.2210,0.0333$, and 0.0181 respectively.

Then weight calculation formula of the event is obtained:

$$
w_{j}=\frac{F_{j}}{\sum_{k=1}^{n} F_{k}}, j=1,2,3 \ldots, n
$$

According to Equation (16), weight based on the information entropy of the four attributes $S S, R$, $t$ and $r$ is $0.0887,0.7393,0.1113$, and 0.0607 .

Finally, the total information entropy $E$ that is an aggregative indicator to estimate the randomness and dispersion of an event is obtained:

$$
E=\sum_{j=1}^{n} w_{j} \cdot E_{j}(j=1,2,3, \ldots, n)
$$

Therefore, total information entropy $E$ of the equivalent structural stress is 0.8294 . Up to this point, fatigue data analysis model based on the information entropy is set up. Fatigue data with all three types of stress based on this evaluation model will be analyzed in the following section. In order to show each step of the evaluation model clearly a flowchart, as shown in Figure 5, is helpful to the following process. 


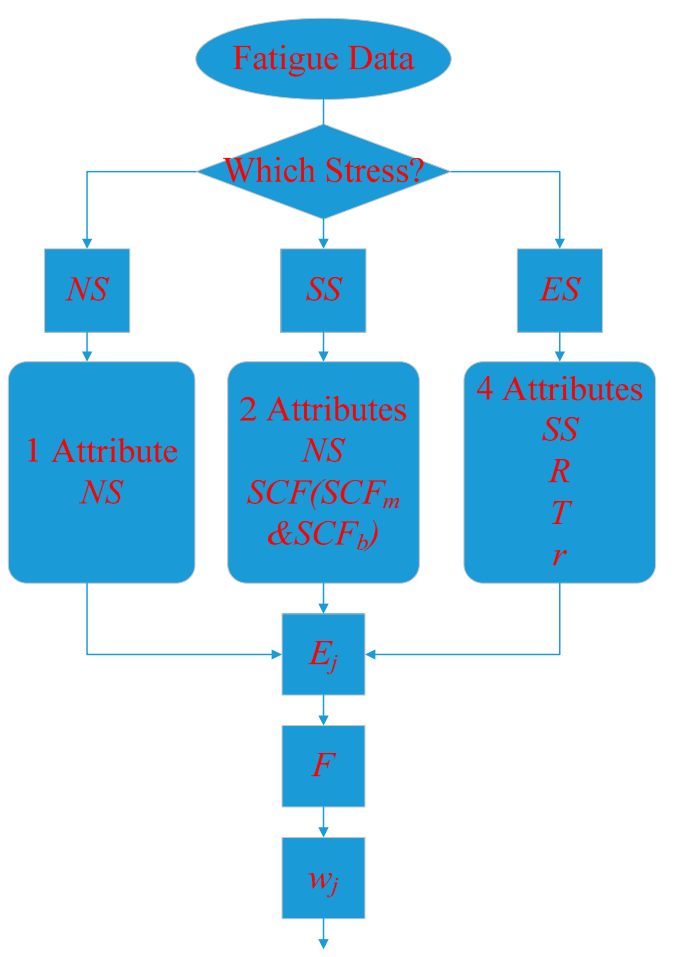

Figure 5. Flowchart of the evaluation model.

\section{Results and Discussion}

On the basis of the evaluation model set up above, a comparison discussion between the standard deviation and total information entropy is carried out. In addition, quantitative contribution of various attributes on the distribution of the S-N curves will be analyzed and discussed in the following content from NS, SS, to ES, respectively. Then the key attribute which affects the S-N curves most can be explored, which is ought to be improved to optimize the master S-N method based on the equivalent structural stress. The results and discussion reveal that the fatigue life evaluation method based on the information entropy can be a credible and effective method of the weld fatigue prediction.

With respect to nominal stress (NS), the property value of itself is the sole attribute to influence the S-N curve distribution of Figure 2 without taking stress concentration, loading mode, and joint type, etc., into account. Therefore, the information entropy weight value of the nominal stress is 1 on the basis of Equation (16). Next, the total information entropy of nominal stress was calculated as 0.9702 that approaches 1, extremely, which indicates that the dispersion degree of the welding fatigue data is great, thus leading to the disorder and non-systematic points of the corresponding S-N curve to some extent. In Figure 2, the total of 48 sets of welding fatigue data scatter out of order and locate mainly from -2 to +2 deviation curve magnifications, which is consistent with the large information entropy value of 0.9702 .

With regard to structural stress (SS), membrane and bending stress concentration factors are taken into consideration, defined as $S C F_{m}$ and $S C F_{b}$. Firstly, the total stress concentration factor (SCF) was obtained by the operation of $S C F_{m}$ plus $S C F_{b}$ so that structural stress can be calculated through multiplying nominal stress by the total stress concentration factor $(S C F)$. As a result, there are three attributes nominal stress, $S C F_{m}$ and $S C F_{b}$ corporately having effects on the distribution of S-N curve in Figure 3. According to the equations from Equations (9)-(17), the information entropy of the $S C F$ were calculated as shown in Table 3 as well as the information entropy of structural stress is shown in Table 4 . The total information entropy of structural stress was 0.8881 , which is $8.46 \%$ smaller than that of nominal stress, resulting in that the distribution areas of the welding fatigue data are much narrower. By contrasting Figures 2 and 3, much more of the welding fatigue data located between 
-1 and +1 deviation curve magnifications, which is in agreement with the $8.46 \%$ decrease of the information entropy.

Table 3. Information entropy of SCF.

\begin{tabular}{ccc}
\hline Information Entropy of $\boldsymbol{S C F}$ & $\boldsymbol{S C F}_{\boldsymbol{m}}$ & $\boldsymbol{S C \boldsymbol { F } _ { \boldsymbol { b } }}$ \\
\hline$E$ & 0.8498 & 0.9709 \\
$F$ & 0.1502 & 0.0291 \\
$w$ & 0.8377 & 0.1623 \\
$E \cdot w$ & 0.7118 & 0.1576 \\
$E_{\text {total }}$ & \multicolumn{2}{c}{0.8694} \\
\hline
\end{tabular}

Table 4. Information entropy of structural stress.

\begin{tabular}{ccc}
\hline Information Entropy of $S \boldsymbol{S}$ & Nominal Stress & SCF \\
\hline$E$ & 0.9702 & 0.8694 \\
$F$ & 0.0298 & 0.1306 \\
$w$ & 0.1856 & 0.8144 \\
$E \cdot w$ & 0.1801 & 0.7080 \\
$E_{\text {total }}$ & \multicolumn{2}{c}{0.8881} \\
\hline
\end{tabular}

When it comes to equivalent structural stress (ES), structural stress (SS), loading ratio $(R)$, plate thickness $(t)$, and bending ratio $(r)$ are taken into account and analyzed, respectively labeled as $S S, R, t$, and $r$ in the context. As discussed before, there is no doubt that the above four attributes affect the stress calculation accuracy and S-N curve distribution to some degree. According to the equations from Equations (9)-(17), the information entropy appeared in Table 5 of the equivalent structural stress is 0.8294 , which is $6.61 \%$ smaller than that of structural stress and $14.51 \%$ smaller than that of nominal stress obviously. Subsequently, the equivalent structural stress rearranges the S-N points much more uniformly near the mean S-N curve, which demonstrates that fatigue data classification based S-N curves can be significantly reduced into possibly a single master S-N curve. The single master S-N curve phenomenon attributes to the smallest information entropy of the three types of stresses.

Table 5. Information entropy of equivalent structural stress.

\begin{tabular}{ccccc}
\hline Information Entropy of $E S$ & Structural Stress & Ratio & Thickness & $r$ \\
\hline$E$ & 0.9735 & 0.7790 & 0.9667 & 0.9819 \\
$F$ & 0.0265 & 0.2210 & 0.0333 & 0.0181 \\
$w$ & 0.0887 & 0.7393 & 0.1113 & 0.0607 \\
$E \cdot w$ & 0.0863 & 0.5759 & 0.1076 & 0.0596 \\
$E_{\text {total }}$ & 0.8294 \\
\hline
\end{tabular}

As mentioned in Section 3.1, the standard deviation of the three S-N curves is 0.5602, 0.2269, and 0.1939 , respectively, and it is obvious that the width between the deviation curves is narrower. On the other hand, the total weighted information entropy of fatigue data based on the three types of stress is $0.9702,0.8881$, and 0.8294 according to the Equations (9)-(17). The standard deviation of the nominal stress is 0.5602 and information entropy of the nominal stress is 0.9702 , both of which are the largest of the three types of stresses. Meanwhile, the data points of Figure 2 based on nominal stress are disordered and non-systematic. The standard deviation of the equivalent structural stress is 0.1939 and information entropy of the equivalent structural stress is 0.8294 , both of which are the smallest of the three types of stresses. A single master S-N curve is obtained by the amendment of the several attributes. From nominal stress to equivalent structural stress, there is consistency between the reducing trend of the weighted information entropy and the smaller and smaller standard 
deviation of the S-N curves, which is shown in Figure 6. Thus, from this perspective, the fatigue life evaluation method based on the information entropy can be a credible and effective method of the weld fatigue prediction.

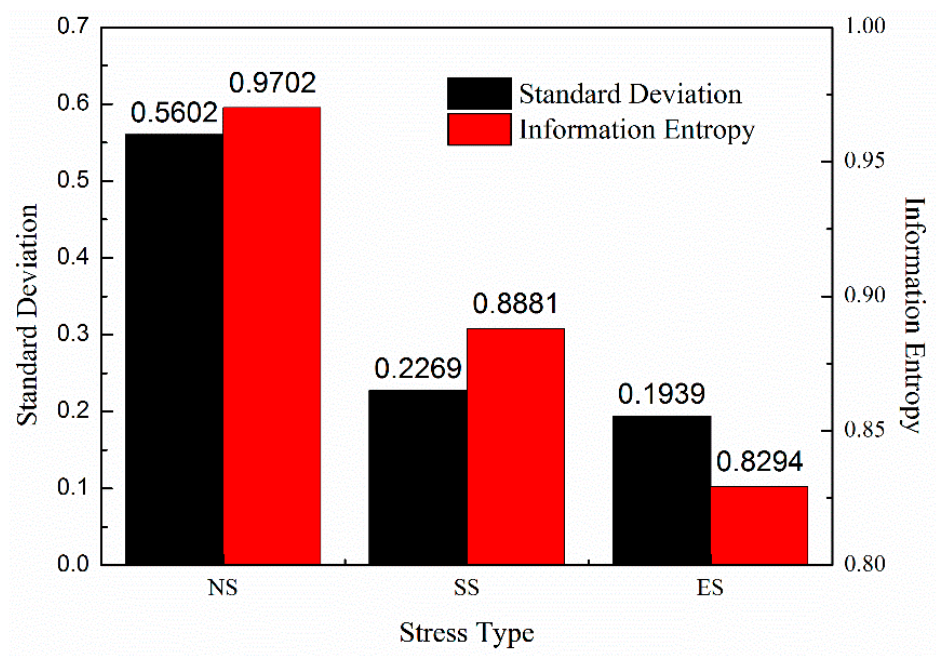

Figure 6. Contrast between the standard deviation and total information entropy.

Next, the attributes that have effects on the distribution of the S-N curves will be analyzed and discussed. There are two main attributes nominal stress and SCF corporately having effects on the distribution of S-N curve based on structural stress. According to Tables 3 and 4 , the information entropy of nominal stress and SCF is 0.9702 and 0.8694 . As known from [8], the fatigue property of welding structure are influenced by all kinds of attributes to different extent, thus, the attributes are supposed to assign in weight value of different levels. Based on the Equation (16), the attributes of nominal stress and SCF possessed weight value of 0.1856 and 0.8144 , respectively, then weighted information entropy of each attribute was obtained as 0.1801 and 0.7080 via multiplying information entropy by weight value. Similarly, weighted information entropy of $S C F_{m}$ and $S C F_{b}$ is 0.7118 and 0.1576. As a result, the three attributes nominal stress, $S C F_{m}$ and $S C F_{b}$ possessed $20.28 \%, 65.27 \%$, and $14.45 \%$ of the total information entropy of structural stress as showed in Figure 7 . For the sector diagram depicting the weighted information entropy of nominal stress, $S C F_{m}$ and $S C F_{b}$ are carried out in Figure 6, in which it is not hard to discover that weighted information entropy of nominal stress is about $20 \%$ so that nominal stress has a little effect on the distribution of structural stress S-N curve in Figure 3. While the weighted information entropy of SCF possesses $79.72 \%$ of the pie diagram, which illustrates that $S C F$ plays a decisive role in forming the S-N curve in Figure 3, between the $S C F_{m}$ and $S C F_{b}, S C F_{m}$ possesses $81.87 \%$ of the $S C F$ and $65.27 \%$ of the total $S C F$ so that $S C F_{m}$ plays an indispensable role in calculating structural stress.

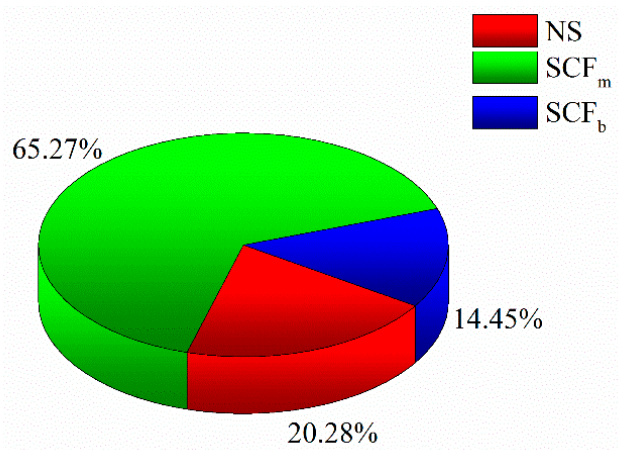

Figure 7. Weighted information entropy of structural stress attributes. 
It is obvious that the weight-based information entropy of the four attributes of ES are respectively $0.0863,0.5759,0.1076$, and 0.0596 via multiplying information entropy by weight value as expressed in Equation (17). The second sector diagram displaying the weight based information entropy of $S S$, $R, t$, and $r$ is drew in Figure 8, in which the value of $r$ is drastically small so that the attribute $r$ has finite effect on the process of establishing the S-N curve. When calculating the $S C F$, some effect of $r$ has been taken into account in the total SCF to consider the stress concentration, the decision-making degree of $r$ is weakened and harmonized in the equivalent structural stress. Additionally, another two important factors, SS and $t$, were then studied. Their weighted information entropy is 0.0863 and 0.1076 , accounting for $10.41 \%$ and $12.97 \%$ of the final total entropy of the equivalent structural stress. Through considering the contribution of SCF and NS, SS has been calculated precisely so that SS just has a little effect on the value of the equivalent structural stress. According to [1], changing of the plate thickness will lead to the stress distribution rule varying, thus plate thickness will affect the correction of the S-N curve to some degree. Finally, the vital attribute $R$ occupies $69.44 \%$ of the total information entropy due to the high weighted information entropy 0.7393 , which proves that stress ratio is a key factor in leading to fatigue destruction.

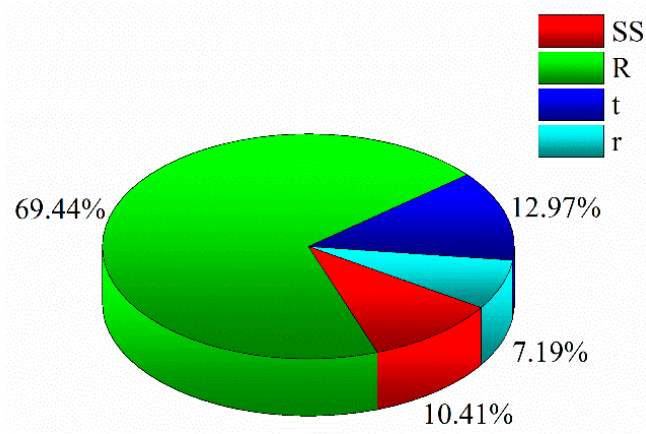

Figure 8. Weighted information entropy of equivalent structural stress attributes.

It is worth noting that all S-N data considered in this paper reflect a broad stress ratio $(R)$ spectrum from 0 to 0.5 . The effects of the $R$ on the S-N data distribution are seen as significant according to the large weight based information entropy. According to [23,24], it is demonstrated that the effects of $R$ become more pronounced as $R$ becomes small, particularly near the threshold regime. On the basis of the two stage crack growth mode, the $R$ effects on the stress intensity factor range can be included either in the first stage of the crack growth or the second stage of the crack growth. Especially, the effects of the stress ratio $R$ is more dominant when a crack is very small, or dominated by the first stage of the crack growth governed by exponent $\mathrm{n}$ in the present two-stage growth mode of Equation (5). It is important to point out that the stress ratio $R$ effects on crack growth rate are much stronger if $R$ is negative than the positive $R$ while there is no negative stress ratio $R$ in the well-documented data used in this paper, which remains to be investigated hereafter.

\section{Conclusions}

Information entropy was firstly imported to study the aluminum alloy welded joint low-cycle fatigue data. S-N distribution of 48 sets of aluminum alloy welding joints were carried out based on nominal stress, structural stress, and the equivalent structural stress, respectively. Fatigue data evaluation model based on information entropy was carried out to study the decision attributes of the three S-N curve distribution method. Some conclusions are addressed as follows:

(1) Three S-N curves for aluminum alloy welded joints are established based on nominal stress, structural stress, and equivalent structural stress by the means of the least square method. The standard deviation of the three S-N curves is $0.5602,0.2269$, and 0.1939 , respectively. On the other hand, the total weighted information entropy of fatigue data based on the three types of 
stress is $0.9702,0.8881$, and 0.8294 . From nominal stress to equivalent structural stress, there is consistency between the reducing trend of the weighted information entropy and the smaller and smaller standard deviation.

(2) Effects of the decision attributes on the distribution of the S-N curves were analyzed and discussed. The total information entropy of structural stress was 0.8881 , which is $8.46 \%$ smaller than that of nominal stress. The three attributes nominal stress, $S C F_{m}$ and $S C F_{b}$ possessed $20.28 \%$, $65.27 \%$, and $14.45 \%$ of the total information entropy of structural stress, of which $S C F_{m}$ plays an indispensable role in calculating structural stress.

(3) The information entropy of the equivalent structural stress is 0.8294 , which is $6.61 \%$ smaller than that of structural stress and $14.51 \%$ smaller than that of nominal stress. The weight based information entropy of the four attributes of $E S$ are respectively $0.0863,0.5759,0.1076$, and 0.0596 . The vital attribute $R$ occupies $69.44 \%$ of the total information entropy, which proves that stress ratio is a key factor in leading to fatigue destruction. With the importance level of the attributes on the S-N curves investigated, the correction of $R$ in the equivalent structural stress based master S-N curve method should be carried out to make the welding fatigue prediction much more accurate.

(4) The fatigue data evaluation model based on information entropy can be a credible and effective model of the weld fatigue prediction.

(5) Information entropy is a probabilistic concept which needs a large amount of experimental data to support, while fatigue experiments are usually expensive and time-consuming. Next, fatigue experiments of various parameters should be carried out to verify the applicability. As well, effects of the negative stress ratio $R$ on the information entropy remains to be investigated.

Acknowledgments: The authors are grateful for the supports by National Science Foundation of China (Project No. 51175054).

Author Contributions: Yaliang Liu realized this work, analyzed thsults and wrote the paper. Li Zou and Yibo Sun contributed to the figures and the paper revision. Xinhua Yang revised the paper finally. All authors have read and approved the final manuscript.

Conflicts of Interest: The authors declare no conflict of interest.

\section{References}

1. Dong, P. A structural stress definition and numerical implementation for fatigue analysis of welded joints. Int. J. Fatigue 2001, 23, 865-876. [CrossRef]

2. Fricke, W. Fatigue analysis of welded joints state of development. Mar. Struct. 2003, 16, 185-200. [CrossRef]

3. BS7608-1993. Fatigue Design and Assessment of Steel Structures; The British Standards Institution: London, UK, 1999.

4. Niemi, E. Stress Determination for Fatigue Analysis Welded Components; Abington Publishing: Cambridge, UK, 1995; pp. 66-69.

5. Sun, Y.; Yang, X. Study on the Correction of S-N Distribution in the Welding Fatigue Analysis Method Based on the Battelle Equivalent Structural Stress by Rough Set Theory. J. Mech. Eng. 2014, 60, 600-606. [CrossRef]

6. Niemi, E. On the Determination of Hot Spot Stresses in the Vicinity of Edge Gussets; Lappeenranta University of Technology, Department of Mechanical Engineering: Lappeenranta, Finland, 1994; Volume 18.

7. Niemi, E.; Tanskanen, P. Hot spot stress determination for welded edge gussets. Weld. World 2000, 44, 31-37.

8. Dong, P.; Hong, J.K.; Osage, D.; Prager, M. Master S-N Curve Approach for Fatigue Evaluation of Welded Components; Welding Research Council: New York, NY, USA, 2002.

9. Li, X.; Li, Q.; He, K.; Khalila, A.H. Arc stability analysis of square wave alternating current submerged arc welding based on wavelet energy entropy. J. Converg. Inf. Technol. 2012, 7, 710-718.

10. Kavrishvili, Z.O.; Chernov, A.V.; Burdakov, S.M. Evaluation of the quality of the components of the process of manual arc welding using entropy methods. Weld. Int. 2007, 21, 754-756. [CrossRef]

11. Amiri, M.; Khonsari, M.M. On the Role of Entropy Generation in Processes Involving Fatigue. Entropy 2012, 14, 24-31. [CrossRef] 
12. Skorobogatov, S.M. The principle of information entropy for the description of fracture at multiple dimensions in real materials (concrete, rock) of buildings and rock seams. Int. J. Fatigue 2004, 128, 335-343. [CrossRef]

13. Guan, X.; Jha, R.; Liu, Y. Probabilistic fatigue damage prognosis using maximum entropy approach. J. Intell. Manuf. 2012, 23, 163-171. [CrossRef]

14. Huang, M.; He, J.; Guan, X. Probabilistic inference of fatigue damage propagation with limited and partial information. Chin. J. Aeronaut. 2015, 28, 1055-1065. [CrossRef]

15. Tucker, J.P.; Chan, D.K.; Subbarayan, G.; Handwerker, C.A. Maximum entropy fracture model and fatigue fracture of mixed $\mathrm{SnPb} / \mathrm{Sn3}$.0Ag5.0Cu Solder alloys. In Proceedings of the 13th IEEE ITHERM Conference, San Diego, CA, USA, 30 May-1 June 2012.

16. Li, H.; Wen, D.; Lu, Z.; Wang, Y.; Deng, F. Identifying the Probability Distribution of Fatigue Life Using the Maximum Entropy Principle. Entropy 2016, 18, 111. [CrossRef]

17. Paris, P.C. Fracture mechanics and fatigue: A history perspective. Fatigue Struct. 1998, 21, 535-540. [CrossRef]

18. The American Society of Mechanical Engineers. ASME Boiler and Pressure Vessel Code Section VIII Division 2 Part 5: Design by Analysis Requirement; ASME: New York, NY, USA, 2007.

19. Núñez, J.A.; Cincotta, P.M.; Wachlin, F.C. Information entropy. Celest. Mech. Dyn. Astron. 1996, 64, 43-53. [CrossRef]

20. Sidhom, N.; Laamouri, A.; Fathallah, R.; Braham, C.; Lieurade, H.P. Fatigue Strength Improvement of 5083 H11 Al-alloy T-welded Joints by Shot Peening: Expermental Characterization and Predictive Approach. Int. J. Fatigue 2005, 27, 729-745. [CrossRef]

21. Beretta, S.; Sala, G. A Model for Fatigue Strength of Welded Lap Joints. Fatigue Fract. Eng. Mater. Struct. 2005, 28, 257-264. [CrossRef]

22. Da Cruz, J.A.M.P.; Costa, J.D.M.; Borrego, L.F.P.; Ferreira, J.A.M. Fatigue Life Prediction in AlMgSi1 Lap Joint Weldments. Int. J. Fatigue 2000, 22, 601-610. [CrossRef]

23. Kujawski, D. A New $\left(\Delta K^{+} K \max \right)^{0.5}$ Driving Force Parameter for Crack Growth in Aluminum Alloys. Int. J. Fatigue 2001, 23, 733-740. [CrossRef]

24. Kujawski, D. Enhanced Model of Partial Crack Closure for Correlation of R-Ratio Effects in Aluminum Alloys. Int. J. Fatigue 2001, 23, 95-102. [CrossRef]

(C) 2017 by the authors; licensee MDPI, Basel, Switzerland. This article is an open access article distributed under the terms and conditions of the Creative Commons Attribution (CC-BY) license (http://creativecommons.org/licenses/by/4.0/). 